\title{
LncRNA TCONS_00004099-derived microRNA regulates oncogenesis through PTPRF in gliomas
}

\author{
Yuhao Wang ${ }^{1 \#}$, Aijun Shan ${ }^{2 \#}$, Zhiwei Zhou ${ }^{3,4,5}$, Wenpeng $\mathrm{Li}^{3,4}$, Lin $\mathrm{Xie}^{4}$, Bo $\mathrm{Du}^{2}$, Bingxi Lei ${ }^{3,4}$ \\ ${ }^{1}$ Nosocomial Infection Control Center, People's Hospital of Shenzhen Baoan District, Shenzhen, China; ${ }^{2}$ Department of Emergency, Shenzhen \\ People's Hospital, The Second Clinical Medical College of Jinan University, The First Affiliated Hospital of Southern University of Science and \\ Technology, Shenzhen, China; ${ }^{3}$ Provincial Key Laboratory of Malignant Tumor Epigenetics and Gene Regulation, Sun Yat-sen Memorial Hospital, \\ Sun Yat-sen University, Guangzhou, China; ${ }^{4}$ Department of Neurosurgery, Sun Yat-sen Memorial Hospital, Sun Yat-sen University, Guangzhou, \\ China; ${ }^{5}$ Faculty of Health Sciences, University of Macau, Macau, China \\ Contributions: (I) Conception and design: B Lei, B Du; (II) Administrative support: B Du, A Shan; (III) Provision of study materials or patients: B Lei, \\ A Shan; (IV) Collection and assembly of data: Y Wang; (V) Data analysis and interpretation: Y Wang; (VI) Manuscript writing: All authors; (VII) \\ Final approval of manuscript: All authors. \\ "Theses authors contributed equally to this work. \\ Correspondence to: Bo Du, PhD, MD. Department of Emergency, Shenzhen People's Hospital, The Second Clinical Medical College of Jinan \\ University, The First Affiliated Hospital of Southern University of Science and Technology, No. 1017, Dongmen North Road, Shenzhen, China. \\ Email: ftipa@szhospital.com; Bingxi Lei. PhD, MD. Department of Neurosurgery, Sun Yat-sen Memorial Hospital, Sun Yat-sen University, 107 \\ Yanjiang West Road, Guangzhou, China. Email: leibingxi@mail.sysu.edu.cn.
}

Background: Glioblastoma is the most common and aggressive primary tumor in the central nervous system (CNS). Patients with glioblastomas have poor prognosis due to its aggressive clinical behavior and resistance to the chemotherapeutic agent temozolomide (TMZ). Aberrant long non-coding RNAs (lncRNAs) are involved in glioma progression and its regulatory mechanisms. Analysis of sequencing data identified a new lncRNA, named lncRNA TCONS_00004099, which could derive a new microRNA and was highly expressed in glioma.

Methods: To elucidate the role of lncRNA TCONS_00004099 in gliomas, Quantitative Real-time PCR (qPCR) was used to assess the differential expression of lncRNA TCONS_00004099 and its related miRNA in glioma tissues, normal brain tissues, glioma cell lines (U87 and U251 cells), and a normal human embryonic brain cell line (HEB). Cell Counting Kit-8 (CCK8) assays to assess cell proliferation, flow cytometry assays examining apoptosis and the cell cycle, colony formation assays, wound healing assay, transwell assays, and zebrafish xenograft models were performed to further clarify the effects of the lncRNA and the related miRNA. Finally, Western blots were carried out to verify the mechanisms related to PTPRF (Protein Tyrosine Phosphatase Receptor Type F).

Results: LncRNA TCONS_00004099 was significantly increased in glioma tissues and glioma cell lines. A novel miRNA (miRNA TCONS_00004099) derived from the lncRNA was identified by qPCR. Knockdown of this lncRNA suppressed cell proliferation, migration, invasion and enhanced TMZ-induced apoptosis in U87 and U251 cell lines in vitro and in vivo. The miRNA mimics or inhibitor of miRNA TCONS_00004099 was used to reverse the effects of knockdown or overexpression of lncRNA TCONS_00004099, respectively. Western Blot analyses verified that PTPRF is one of the downstream targets of lncRNA TCONS_00004099. Conclusions: These results demonstrated that lncRNA TCONS_00004099 promoted malignant behaviors in gliomas, including proliferation, metastasis, and anti-apoptosis. The effect of lncRNA TCONS_00004099 was mediated through miRNA TCONS_00004099 and its target PTPRF. Thus, the lncRNA TCONS_00004099/miRNA/PTPRF axis may be a potential therapeutic target for gliomas.

Keywords: Glioma; lncRNA TCONS_00004099; microRNA; PTPRF

(c) Annals of Translational Medicine. All rights reserved. 
Submitted Feb 26, 2021. Accepted for publication Jun 17, 2021.

doi: $10.21037 /$ atm-21-2442

View this article at: https://dx.doi.org/10.21037/atm-21-2442

\section{Introduction}

Glioma derived from the neural ectoderm is the most common type of primary malignant brain cancer and accounting for $50 \%$ of primary brain malignancies(1). The poor prognosis in patients with glioma is due in part to the strong tumor growth capacity of cancer cells and the tumor's resistance to radiotherapy and chemotherapy induced apoptosis (2). As a chemotherapeutic agent, temozolomide (TMZ) is a first-line therapy for gliomas, especially in glioblastoma multiforme (GBM), and has achieved remarkable success (3). However, a large proportion of patients develop tumor resistance to TMZ due to the uncontrolled proliferation of glioma cells and tumor growth (4). Therefore, in addition to understanding the normal oncological behaviors of gliomas (cell viability, proliferation, invasion, apoptosis, and cell cycle), it is also important to explore the manifestations of chemoresistance.

Long non-coding RNA (lncRNA) is a section of noncoding RNA greater than 200 nucleotides with no potential to translate into protein (5). The functions of lncRNAs have been associated with microRNAs (miRNAs) and have been shown to be involved in gene regulation of gliomas (6). As a regulatory molecule, lncRNAs have diverse functions in cell cycle, proliferation, invasion and survival $(7,8)$. Moreover, it was shown that lncRNAs in gliomas has high rates of chemoresistance and recurrence (9-11). Therefore, revealing the mechanisms that mediate the chemoresistance of gliomas is urgently needed. Analysis of sequencing data identified a new lncRNA, named lncRNA TCONS_00004099, which could derive a new microRNA and was highly expressed in glioma. The sequence of the new miRNA is "UGUGGUUCUGGGGCCAUUGUU". Bioinformatics analysis shows that $\operatorname{lncRNA}$ TCONS_00004099 can target protein mRNA depending on the new microRNA which derives from such lncRNA via miRNA binding site prediction software miRanda. 21 nucleotides generated by lncRNA TCONS_00004099 splicing is predicted to bind to PTPRF mRNA and inhibit the translation.

miRNAs are non-coding single-stranded RNA with 18 to 24 nucleotides. miRNAs inhibit the translation of target mRNAs by binding to the 3'-untranslated region (3' UTR) of different target mRNAs (12). In recent years, hundreds of different miRNAs have been identified in cancers. For example, the STAT3/miR-182-5p/PCDH8 axis has been shown to play a key role in glioma tumorigenesis (13). However, the role of miRNAs derived from lncRNA in glioma oncogenesis is still not clear.

Protein tyrosine phosphatase (PTP) works with protein tyrosine kinases to regulate many important signaling pathways in cancer (14). The PTP family constructed of 107 members which divided into 4 subfamilies. PTPRF belongs to class I PTP and is closely related to PTPRD and PTPRS (15). Previous research has shown that MicroRNA-24 enhances breast cancer invasion and metastasis by targeting PTPN9 and PTPRF to promote EGF signaling (16). However, a systematic understanding of how lncRNA contributes to PTPRF in gliomas is lacking. Hence, there is an urgent need to identify novel PTPRFrelated lncRNA and investigate their potential mechanisms of oncogenesis in gliomas.

In this study, we investigated the role and the mechanism of lncRNA TCONS_00004099 in the oncogenesis of gliomas. Moreover, we determined whether lncRNA TCONS_00004099 regulated the migration, invasion, tumor growth, and TMZ resistance of glioma through generating microRNAs. Importantly, we determined that lncRNA TCONS_00004099 promotes the oncogenesis of glioma by generating microRNA and targeting PTPRF.

We present the following article in accordance with the ARRIVE reporting checklist (available at https://dx.doi. org/10.21037/atm-21-2442).

\section{Methods}

\section{Clinical samples and cell lines}

Human glioma samples were obtained from the Neurosurgery Department of the Sun Yat-sen Memorial Hospital, Sun Yat-sen University. Different grades of glioma tissues were obtained from different glioma patients. Normal brain tissues were obtained from patients who required partial brain excision such as patients with traumatic brain injury. All tissues were preserved in liquid nitrogen for subsequent total RNA extraction. The study 
was approved by institutional ethics board of Sun Yat-sen Memorial Hospital, Sun Yat-sen University (No.: 2019060) and conformed to the guidelines of the Declaration of Helsinki (as revised in 2013). Written informed consent was obtained from the patients before the start of the study. The U87 and U251 glioma cell lines, and the HEB cell line were purchased from Shanghai Institutes for Biological Sciences of Chinese Academy of Sciences (SIBS, Shanghai) and cultured in Dulbecco modified Eagle medium (DMEM, GIBCO) supplemented $10 \%$ fetal bovine serum (FBS, GIBCO). The glioma cells were incubated in $37^{\circ} \mathrm{C}$ in humid atmosphere with $5 \% \mathrm{CO}_{2}$.

\section{Oligonucleotide synthesis and transfection, lentivirus vector transfection}

Cells were transfected with lentivirus vector pZHLVCMV-zsGreen 1-puro packaged with the lncRNA TCONS_00004099 overexpression sequence or short hairpin RNA (shRNA). The synthetic inhibitor or mimics of miRNA derived from lncRNA TCONS_00004099, and small interfering RNA (siRNA) were transfected into cell lines using Lipofectamine 3000 (Thermo, MA, USA). All the oligonucleotides and scramble sequences were designed and provided by GenePharma (Shanghai, China). Lentivirus vector pZHLV-CMV-zsGreen1-puro packaged by DongBio.Co.Ltd (Shenzhen, China). The sequences of lncRNA TCONS_00004099 and the related miRNA were demonstrated in Supplementary file.

\section{$R N A$ extraction and $q P C R$}

RNA was isolated from tissues and cells using the Trizol Reagent (TAKARA, Japan). The total RNA was reverse transcribed into cDNA using the Master Mix kit (TAKARA, Japan). Then, the Mir-X miRNA First-Strand Synthesis Kit (TAKARA, Japan) was used in the transcription of miRNA. Quantitative Real-time PCR (qPCR) was carried out with SYBR-Green Master Kit (TAKARA, Japan). The expression levels of target genes were normalized to glyceraldehyde 3-phosphate dehydrogenase (GADPH). The forward primer sequence of LncRNA TCONS_00004099 was 5'-CCTAACAGGTGCTGTGGCA-3', while the reverse sequence was 5'-TCATCGTTGCAGAACAATGGC $-3^{\prime}$. The forward primer sequence of GAPDH was 5'-GCTCTCTGCTCCTCCTGTTC-3', while the reverse primer sequence was 5'-AAATGAGCCCCAGCCTTCTC-3'.

\section{Cell Counting Kit 8 (CCK8) assay}

Cells were harvested and inoculated into 96-well plate (3,000-6,000 cells/per well) and cultured for 24 hours. After treatment, the culture medium was mixed with the CCK8 solution (the proportion was $1: 10$ ) and added to each well. Cells were incubated at $37^{\circ} \mathrm{C}$ for $1-2$ hours. Thereafter, the absorbance of each well was measured at a wavelength of $450 \mathrm{~nm}$.

\section{Wound healing assay and transwell test}

Glioma cells were cultured in six-well plates under different treatment conditions. After 72 hours, the culture media was removed, serum-free medium was added to treat the cells for another 12 hours. A straight wound was scratched using a $200 \mu \mathrm{L}$-pipette tip. The cells would migrate through the scratch and heal the wound. Photographs were taken using an inverted microscope at 0,12 , and 24 hours.

To further detect the migration and invasion ability, transwell assays were performed using transwell inserts (Costar, USA) coated with $50 \mu \mathrm{L}$ Matrigel Matrix (BD, USA). The inserts were put into 24-well plates. Cells in FBS-free DMEM were seeded into the upper chambers while the lower chambers were filled with DMEM with $10 \%$ FBS. After 24 hours, the excess cells were wiped off with a cotton swab. $4 \%$ paraformaldehyde were used to fixed chambers and then stained with $0.1 \%$ crystal violet dye. Cells were counted with an inverted microscope (Olympus).

\section{Colony formation assays}

Glioma cells were cultured in six-well plates under different treatment conditions for 48 hours. $4 \%$ paraformaldehyde were used to fixed cells and then stained with $0.1 \%$ crystal violet dye. The number of colonies per well was counted with an inverted microscope (Olympus).

\section{Apoptosis applications}

Cells were cultured and treated in combination with $300 \mu \mathrm{M}$ temozolomide (TMZ). After 72 hours, cells were digested, centrifuged, and washed with PBS. Binding buffer $(200 \mathrm{~mL})$ and Annexin V $(5 \mu \mathrm{L})$ from the Annexin V/PI Kit (BD, USA) were added to the cells and incubated in the dark for 15 minutes. Thereafter, $10 \mathrm{~mL}$ propidium iodide (PI) was added and flow cytometry was performed 
immediately at 488 and $594 \mathrm{~nm}$.

\section{Western blot analysis}

Protein concentrations were determined using the G250 assay (Keygen). Proteins were separated using sodium dodecyl sulfate polyacrylamide gel electrophoresis (SDS-PAGE) by electrophoresis, then transferred to polyvinylidene difluoride (PVDF) membranes. A PTPRF monoclonal antibody (rabbit; 1:2,000; Abcam, UK) were incubated with the PVDF membranes overnight, followed by incubation with an anti-rabbit secondary antibody (1:10,000; Proteintech, USA). The immune complexes were detected using the Two-color Infrared Laser Imaging System (Odyssey).

\section{Zebrafish xenograft model}

The Zebrafish xenograft model is a one-way trial that transgenic A/B zebrafish line (Zebrafish Technology Platform, Sun Yat-sen University) was used in this study. Zebrafish embryos were raised, staged, and maintained according to standard procedures. Zebrafish embryos (no gender distinction) were anesthetized with $0.02 \%$ tricaine (Sigma) and positioned on a $10-\mathrm{cm}$ Petri dish coated with $3 \%$ agarose. Transfected cells (approximately 300 cells) were injected into the yolk sac of zebrafish embryos 2 days-post fertilization using a Picospritzer (Parker Inc.). After 24 hours, zebrafish were included in the study if the fluorescence foci in the yolk sacs was similar. If any zebrafish had less fluorescence or no fluorescence foci than the other groups, they were not included in the study. After implantation with transfected glioma cells, zebrafish embryos (including non-implanted controls) were maintained at $33{ }^{\circ} \mathrm{C}$ for the next 96 hours. 10 embryos were included for each cell line or condition and a total of 60 embryos were included in each experiment, zebrafish embryos were placed in 96-well plates and randomly divided into each treatment group using a randomization table (http://www.graphpad.com/quickcalcs/randomN2/). For each zebrafish, three different investigators were involved as follows: a first investigator administered the transfected cell. This investigator was the only person aware of the treatment group allocation. A second investigator was responsible for the injected procedure, whereas a third investigator monitored the fluoresce in the yolk sac.

A protocol was prepared before the study without registration. Experiments were performed under a project license (No.: LL-KY-2019105) granted by Institute Ethics Committee, Shenzhen People's Hospital, in compliance with China national or institutional guidelines for the care and use of animals.

\section{Statistical analysis}

For unpaired samples, Student's $t$-test was used to analyze statistical differences. For multiple comparisons with one control group, an analysis of variance followed by the Dunnett test was performed. A P value less than 0.05 was considered statistically significant. All results were independently repeated at least three times.

\section{Results}

\section{LncRNA TCONS_00004099 was upregulated in glioma tissues and cell lines, while knock down LncRNA TCONS_00004099 downregulated glioma cells viability, invasion, and migration in vitro}

The expression levels of lncRNA TCONS_00004099 in different GBM tissues were assessed using qPCR. The levels of lncRNA TCONS_00004099 were markedly upregulated in GBM tissues compared to normal brain tissues. In addition, IncRNA TCONS_00004099 expression was remarkable higher in the U87 and U251 glioma cell lines compared to the HEB cell line (Figure 1A). These results suggested that lncRNA TCONS_00004099 is positively correlated with human glioma.

To knock down lncRNA TCONS_00004099, five siRNAs that interfered with the lncRNA in U87 and U251 cells were assessed. The qPCR results showed that siRNA1 and siRNA2 downregulated the levels of lncRNA TCONS_00004099 most significantly (Figure 1B). The sequence of siRNA1 and siRNA2 is selected for further experiment. CCK8 assays showed that cellular viability was suppressed markedly by siRNA1 and siRNA2 (Figure 1C). Transwell migration tests, transwell invasion assays, and wound healing assays showed that siRNA1 and siRNA2 inhibited the migration and invasion of U87 and U251 cells (Figure 1D). Furthermore, 300 $\mu \mathrm{M}$ temozolomide (TMZ) was used to generate an apoptosis model, and the results demonstrated that knockdown of lncRNA TCONS_00004099 significantly enhanced apoptosis in U87 and U251 cells compared to control cells (Figure 1E).

These data suggested that IncRNA TCONS_00004099 expression was increased in glioma tissues and glioma cell 

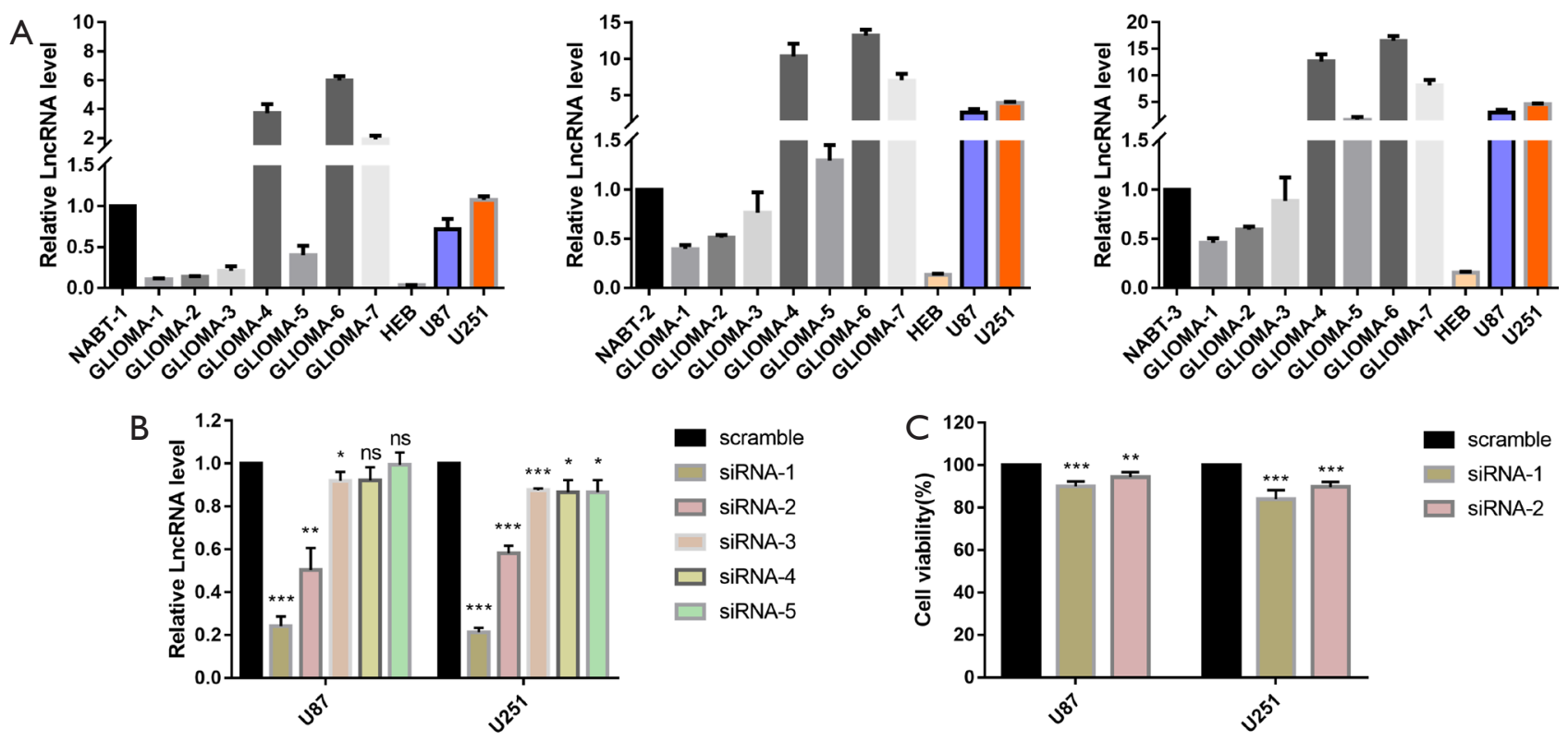

D

U87

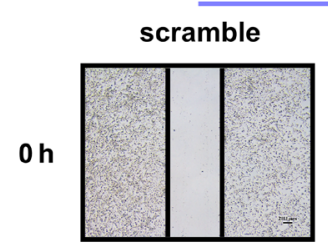
siRNA-1 siRNA-2
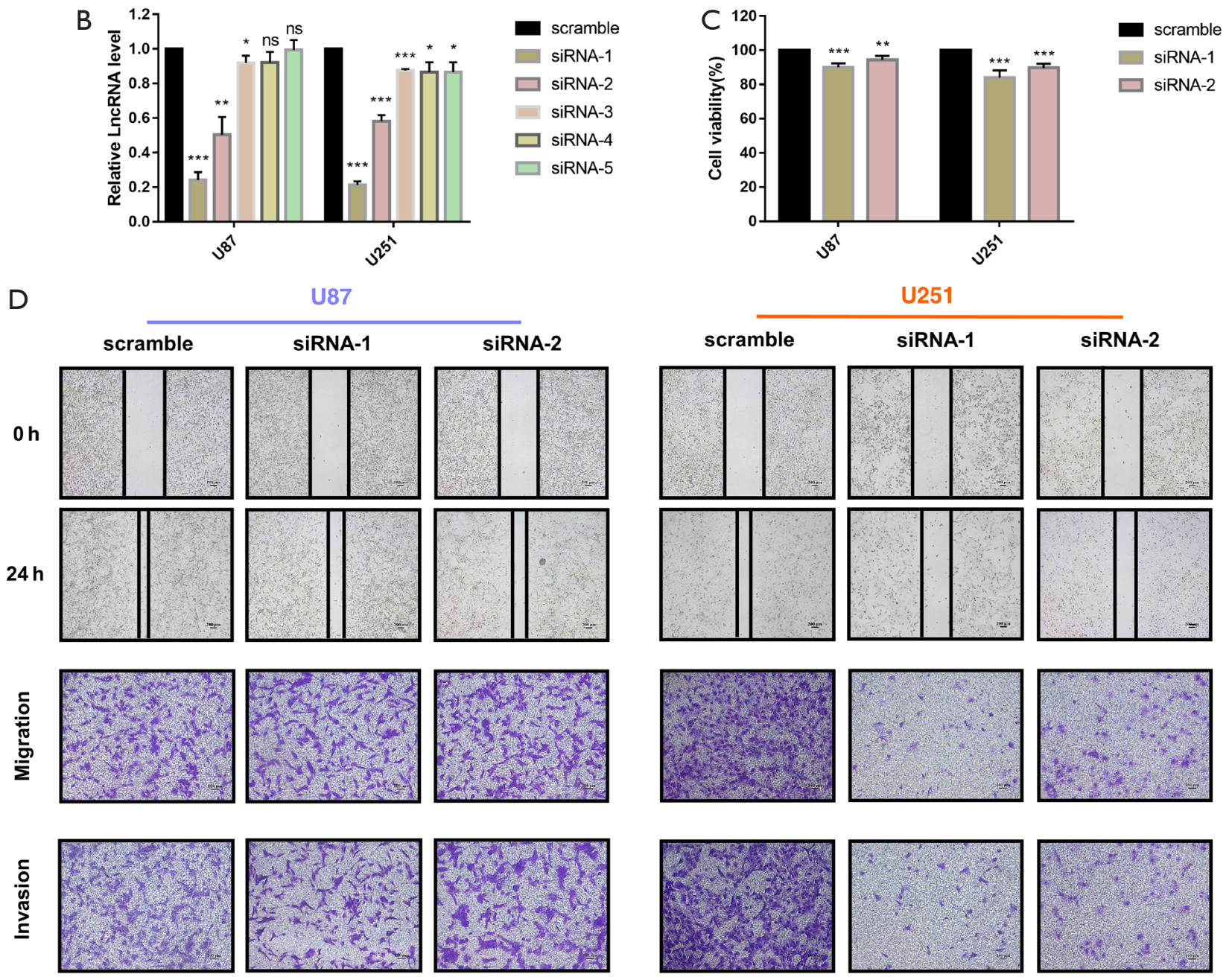

U251
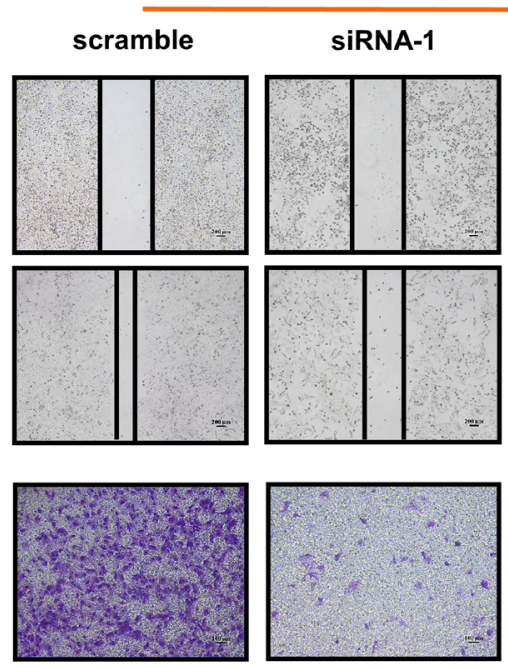

SiRNA-2
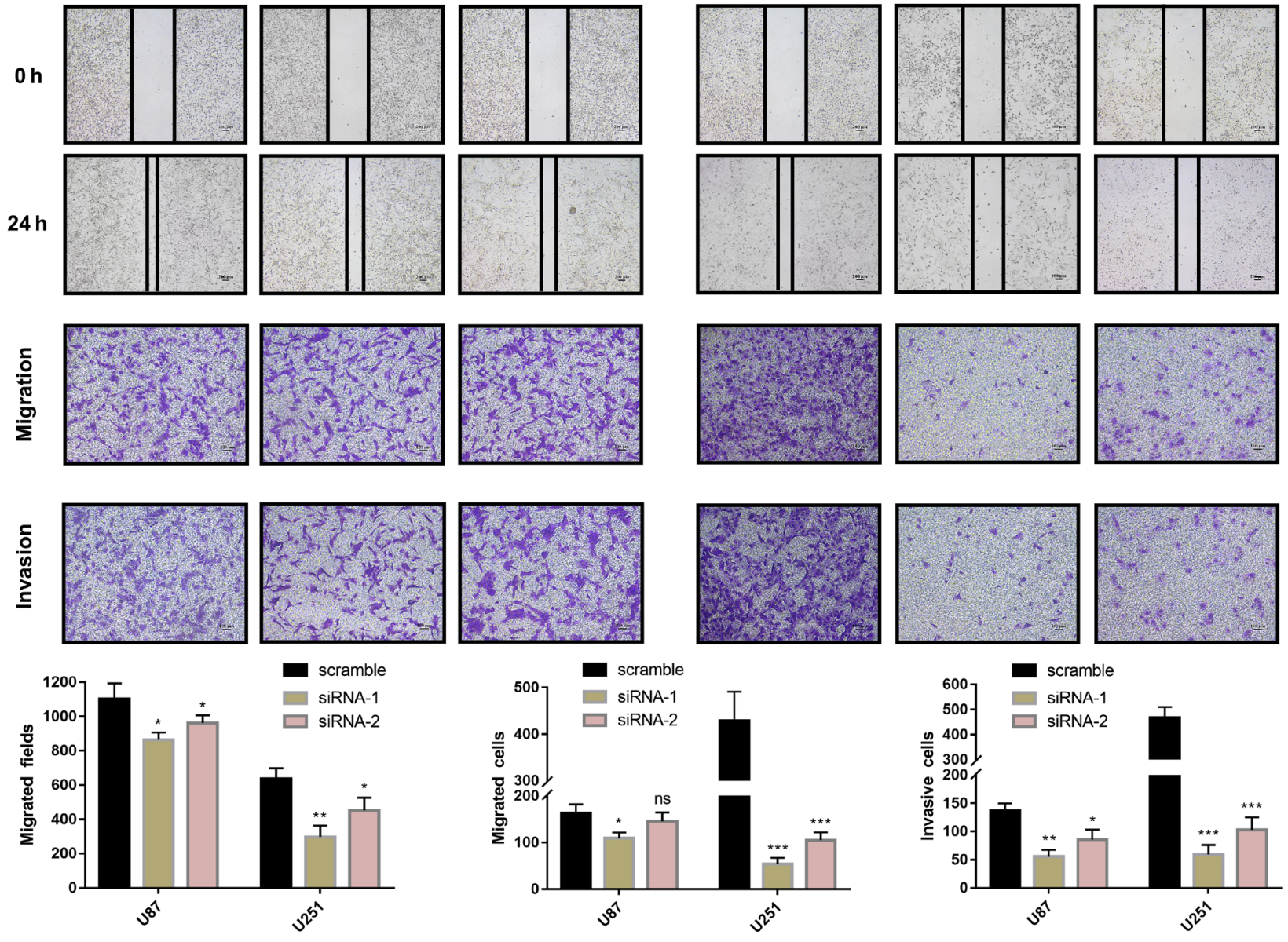
E

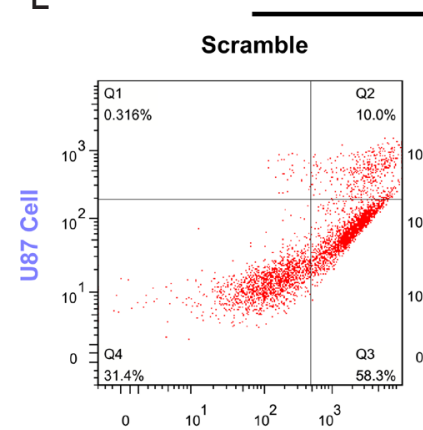

Temozolomide

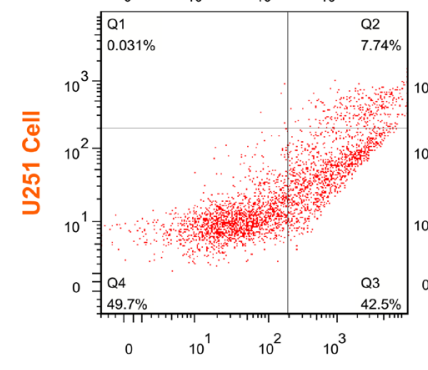

\begin{abstract}
SIRNA-1
\end{abstract}
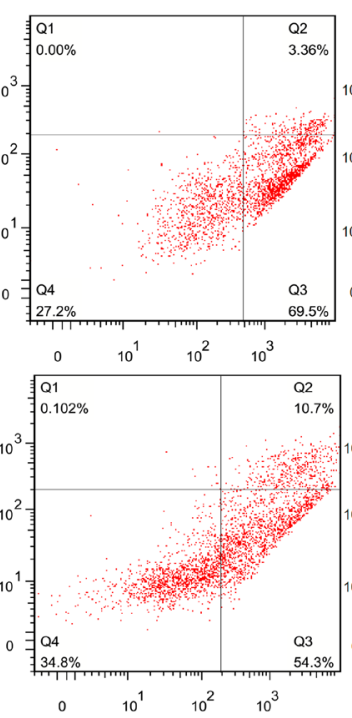
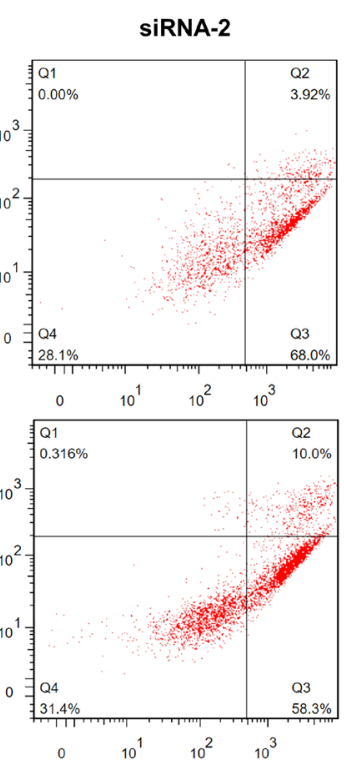
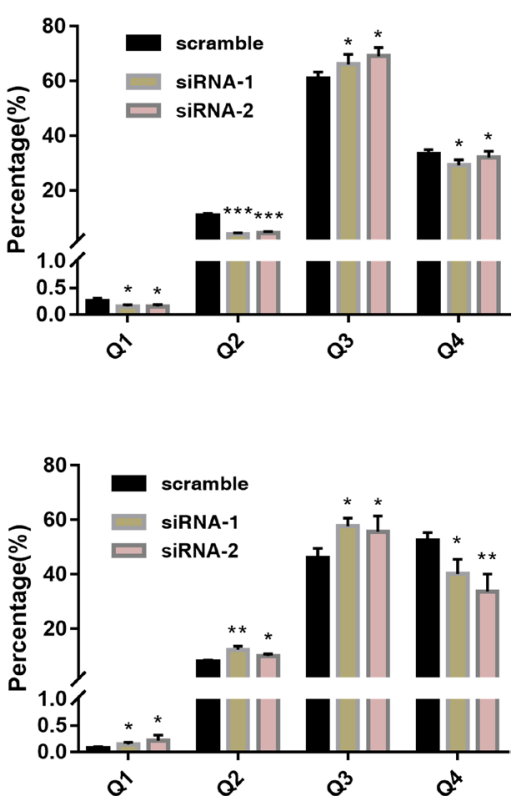

Figure 1 The expression, siRNA interference efficiency and effects of the lncRNA TCONS_00004099 on cells viability, migration, invasion and TMZ sensitivity. (A) The expression of lncRNA TCONS_00004099 in glioma specimens, normal brain tissues, the U87 and U251 glioma cell lines, and the HEB cell line; (B) the expression of lncRNA TCONS_00004099 in U87 and U251 cells after treatment with the 5 different small interfering RNAs (siRNAs) was detected by qPCR; (C) cell Counting Kit-8 was used to analyze the cell viability of U87 and U251 cells after treatment with the siRNA1 and siRNA2; (D) wound healing assay in U87 and U251 cells treated with siRNA1 and siRNA2 for 24 hours (upper, scale bars, $200 \mu \mathrm{m}$ ), transwell migration assays (middle) and transwell invasion assays (underside, scale bars, $100 \mu \mathrm{m}$ ) were used to examine the effects of lncRNA TCONS_00004099 downregulation on migration and invasion ability in U87 and U251 cells. The means of relative migration distance and invaded cells per field are presented, cells were stained with $0.1 \%$ crystal violet solution. The vertical coordinates from left to right are in microns and the number of migrated cells; (E) flow cytometry was used to detect cellular apoptosis in U87 and U251 cells treated with siRNA1 and siRNA2 and temozolomide, statistical analysis of apoptosis in U87 and U251 cells. Scale bars, $100 \mu \mathrm{m}$. Data are expressed as mean $\pm \mathrm{SEM}$. ns, not statistically significant; ${ }^{*}, \mathrm{P}<0.05 ;{ }^{* *}, \mathrm{P}<0.01 ;{ }^{* * *}, \mathrm{P}<0.001$.

lines. Downregulation of IncRNA TCONS_00004099 significantly decreased cell growth, migration, and invasion, and enhanced TMZ-induced apoptosis.

\section{LncRNA TCONS_00004099 enbanced glioma cell viability and proliferation through generating miRNA TCONS_00004099 and targeting PTPRF}

Glioma cell lines were transfected with the overexpression lentivirus vector or shRNA lentivirus vector, which upregulated or downregulated the levels of IncRNA TCONS_00004099, respectively. The upregulated-lncRNA group was named as the overexpression group (OE) while the downregulated-lncRNA group was named as the lowexpression group (LE). The expression levels in each group were confirmed by qPCR assay (Figure $2 A$ ).
Specific qPCR was performed to identify cellular miRNAs and a new miRNA derived from lncRNA TCONS_00004099 named miRNA TCONS_00004099 was identified. The miRNA inhibitor or mimics was transfected into cells to reverse the effects of the lncRNA. The 7 experimental groups included scramble sequences group(scramble), miRNA inhibitor group (miR-IH), miRNA mimics group (miR-MI), lncRNA overexpression group (OE), lncRNA expression plus miRNA inhibitor group $(\mathrm{OE}+\mathrm{IH})$, lncRNA low-expression group (LE), and IncRNA low-expression plus miRNA mimics group (LE + MI). Assessing the miRNA TCONS_00004099 levels revealed that both IncRNA TCONS_00004099 and the miRNA mimics increased the levels of cellular miRNA TCONS_00004099 (Figure 2B).

CCK8 assays demonstrated that the effects of miRNA 


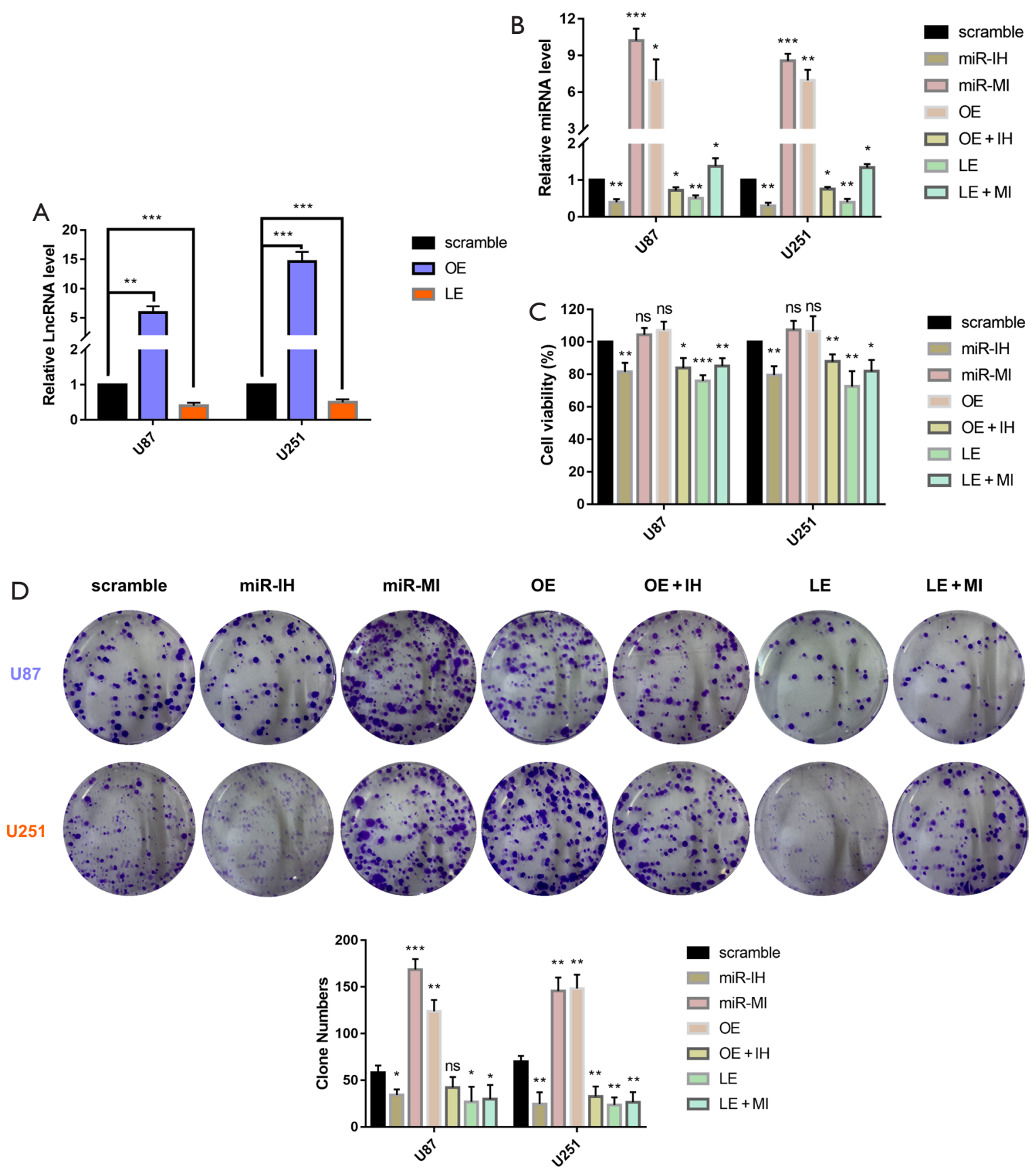

Figure 2 Construction of U87 and U251 cells with stable overexpression and knockdown of lncRNA TCONS_00004099, exploration of the derived miRNA and the effects of proliferation. (A) Quantitative PCR was used to detect the expression of lncRNA TCONS_00004099 in U87 and U251 cells; (B) the miRNA TCONS_00004099 levels in U87 and U251 cells under different treatments, namely, negative control (NC), miRNA TCONS_00004099 inhibitor (miR-HI), miRNA TCONS_00004099 mimics (miR-MI), lncRNA TCONS_00004099 overexpression (OE), lncRNA TCONS_00004099 overexpression plus miRNA TCONS_00004099 inhibitor (OE + IH), lncRNA TCONS_00004099 knockdown (LE), and lncRNA TCONS_00004099 knockdown plus miRNA TCONS_00004099 mimics (LE + MI); (C) the Cell Counting Kit-8 was used to analyze the cell viability of U87 and U251 cells under different treatment conditions; (D) plate colony formation assays were performed to assess the effects of proliferation in U87 and U251 cells under different treatment conditions, cells were stained with $0.1 \%$ crystal violet solution. Magnification $1 \times$. The vertical coordinates are in numbers. Statistical analysis of the colony formation assay in $\mathrm{U} 87$ and $\mathrm{U} 251$ cells. ns, not statistically significant; ${ }^{*}, \mathrm{P}<0.05 ;{ }^{* *}, \mathrm{P}<0.01 ;{ }^{* *}, \mathrm{P}<0.001$. 
TCONS_00004099 on cell viability corresponded to that of IncRNA TCONS_00004099. Overexpression of the lncRNA significantly promoted cell growth and this was reversed by the miRNA inhibitor. Downregulation of IncRNA suppressed cell growth and this was reversed by the miRNA mimics (Figure 2C). Colony formation assays revealed similar results on cell proliferation in both U87 and U251 cell lines (Figure 2D).

Wound healing assays, transwell migration assays, and transwell invasion assays also demonstrated that overexpression of lncRNA TCONS_00004099 enhanced migration and invasion, while knockdown lncRNA decreased the capacity of migration and invasion of U87 and U251 cell. These effects were reversed by both the miRNA inhibitor and the miRNA mimics (Figure $3 A, B$ ).

Glioma Cells in the OE and LE group were injected into the zebrafish tumor model and the invasive ability was assessed by counting the number of fluorescent foci at 96 hours after injection. The results were similar to that observed in vitro (Figure 3C).
Moreover, Flow cytometry assays revealed that lncRNA TCONS_00004099 significantly decreased cell apoptosis and enhanced cell proliferation. In contrast, downregulation of lncRNA TCONS_00004099 decreased proliferation and promoted cell apoptosis in U87 and U251 cells (Figure 4A,B).

Then, we analyzed potential targets of miRNA TCONS_00004099. There were a great number of candidates potentially targeted by miRNA TCONS_00004099, then we finally focused on PTPRF. The potential binding sites appeared nucleotides 6827 to 6837 for PTPRF mRNA (NM_002840) (Figure 4C). Meanwhile, Western blot analyses were used to detect the expression of the miRNA TCONS_00004099targeted PTPRF in U87 and U251 cells. Overexpression of lncRNA TCONS_00004099 suppressed PTPRF expression in U87 and U251 cells, while knockdown of lncRNA TCONS_00004099 increased PTPRF expression in U87 and U251 cells (Figure 4D). These results showed that LncRNA TCONS_00004099 enhanced the

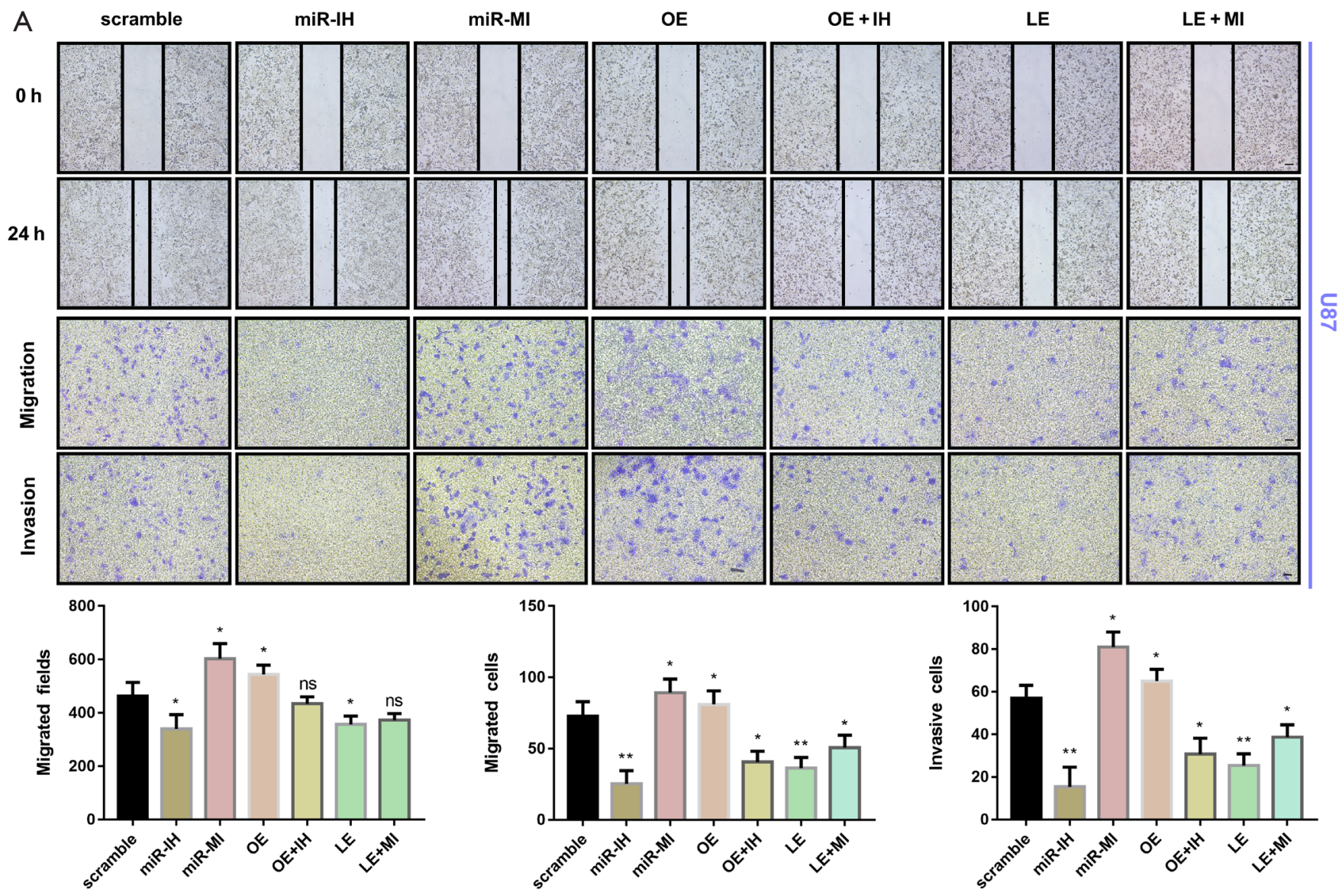




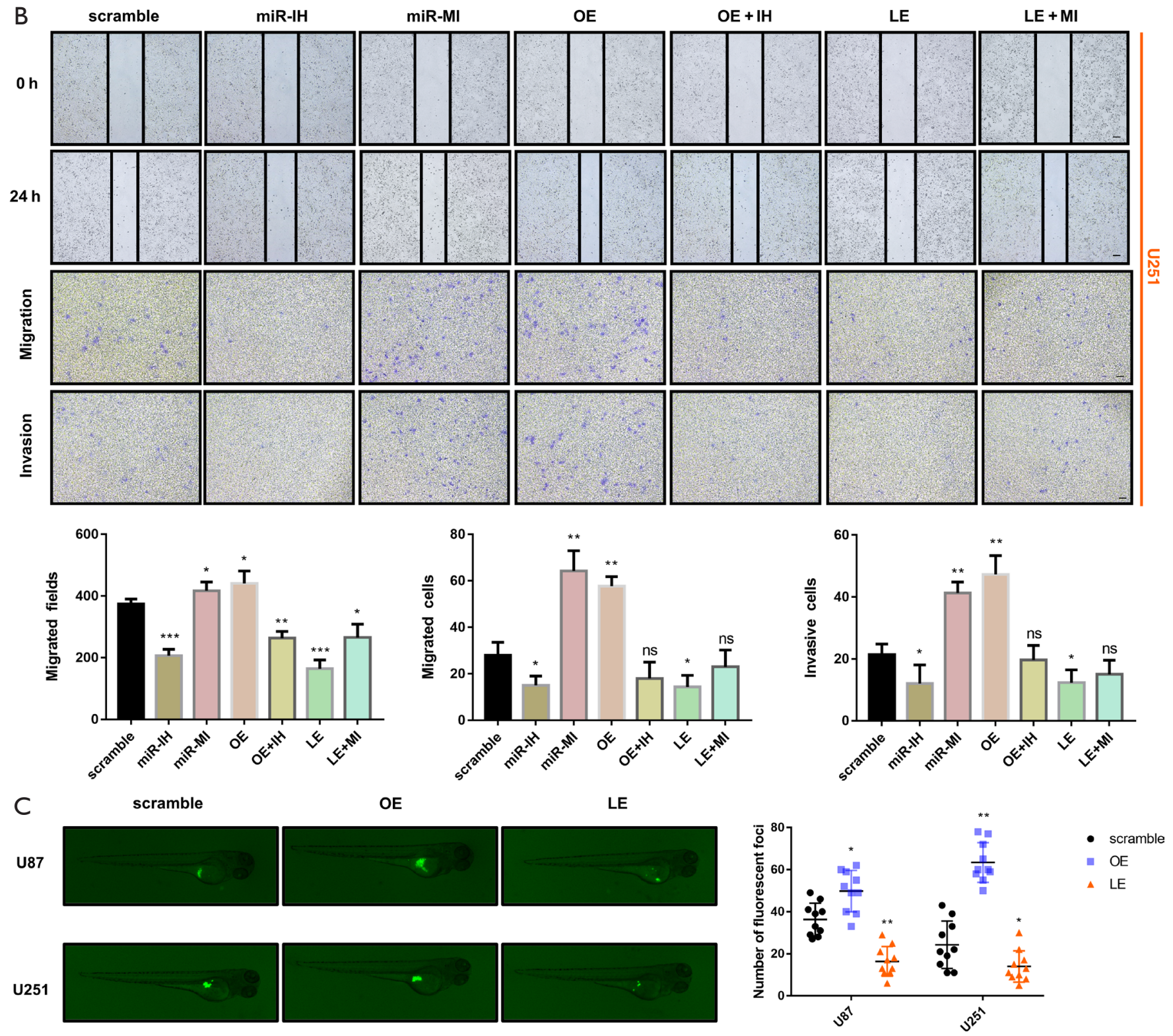

Figure 3 The effects of the lncRNA/miRNA TCONS_00004099 on migration, invasion and zebrafish xenograft model of glioma cells. $(\mathrm{A}, \mathrm{B})$ Wound healing assays $(100 \times)$, transwell migration assays (200x) were used to examine cell migration ability, and transwell invasion assays (200x) were used to examine the invasion ability of U87 and U251 cells after different treatments, cells were stained with $0.1 \%$ crystal violet solution. The means of relative migration distance and invaded cells per field are presented. The vertical coordinates from left to right are the number of migrated cells. (C) The zebrafish xenograft model was used to analyze the invasive ability of U87 and U251 cells after lncRNA TCONS_00004099 overexpression and knockdown, n=10. Fluorescent foci numbers were determined at 96 hours after cells were injected into the yolk sacs of zebrafish. Magnification $3 \times$. The vertical coordinates are the number of fluorescent foci. Data are expressed as mean \pm SEM. ns, not statistically significant; * $\mathrm{P}<0.05 ;{ }^{* *}, \mathrm{P}<0.01$; ***, $\mathrm{P}<0.001$. 

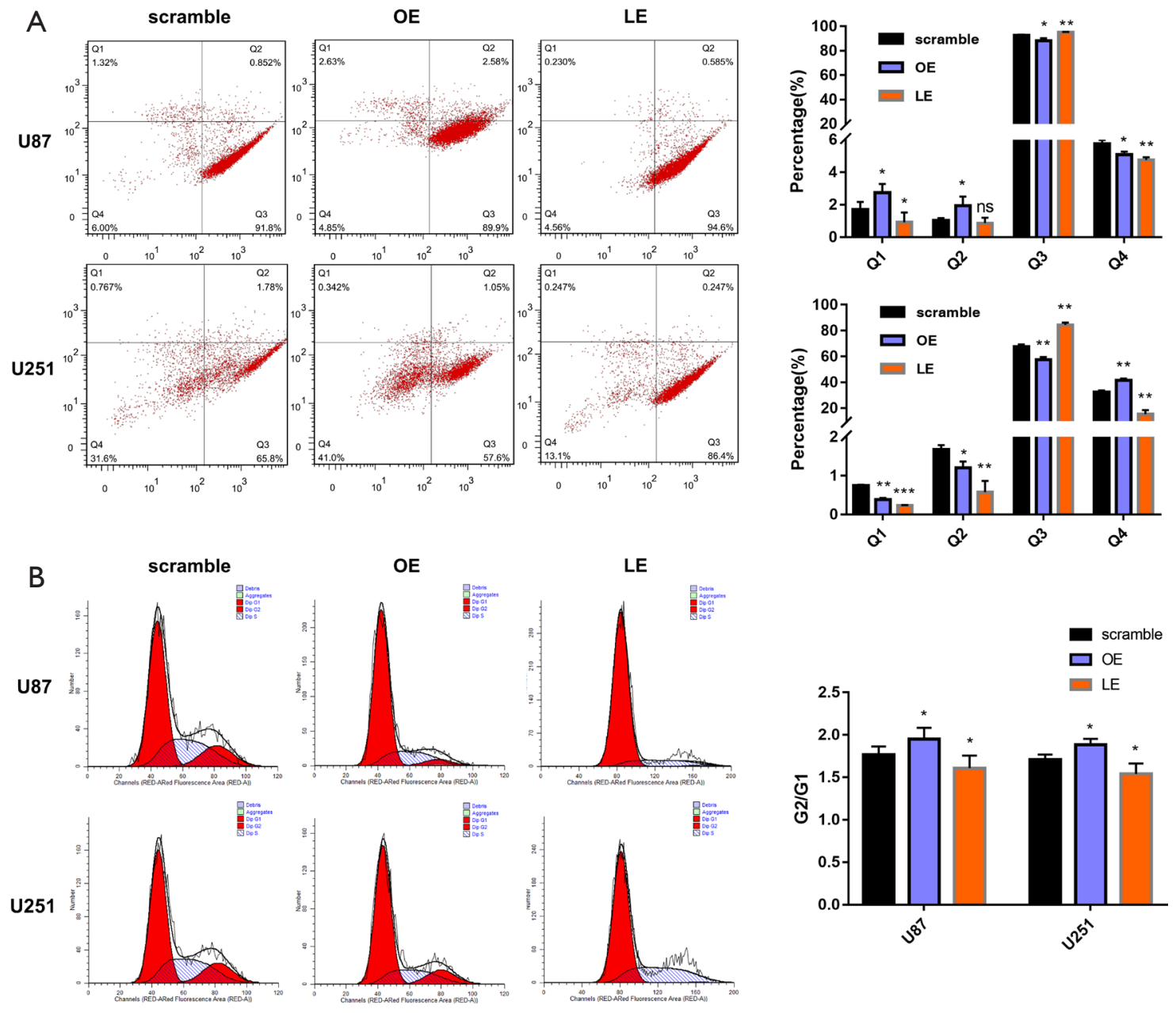

C miRnA TCONS_00004099 3' uugUUACCGGGGUCUUGGUGu 5'

|||||||||| $\mid$

PTPRF $\quad 3^{\prime}$ cat $\mathrm{t} \mathrm{c}$ a $\mathrm{c}$ a gCCCAGAACCACa $5^{\prime}$

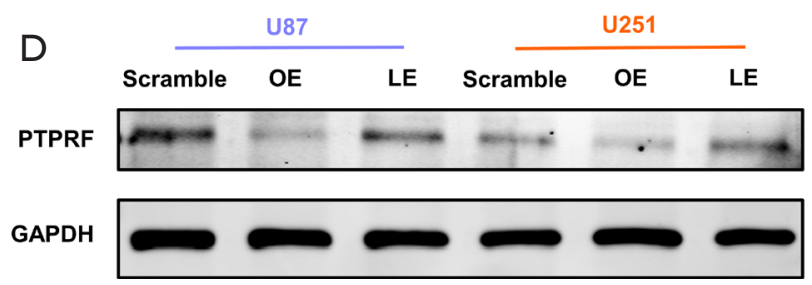

Figure 4 The effects of lncRNA TCONS_00004099 overexpression and knockdown on apoptosis, cell cycle and target protein PTPRF in glioma cells. (A) Apoptosis was analyzed by flow cytometry using U87 and U251 cells in which lncRNA TCONS_00004099 was overexpressed or knocked down. Statistical analysis of apoptosis in U87 and U251 cells; (B) the cell cycle was analyzed by flow cytometry using U87 and U251 cells in which lncRNA TCONS_00004099 was overexpressed or knocked down. Statistical analysis of cell cycle detection in U87 and U251 cells; (C) sequences showing potential miRNA TCONS_00004099 binding sites in PTPRF mRNA nucleotides 6827 to 6837 for PTPRF mRNA (NM_002840); (D) Western blot was used to detect the expression of the miRNA TCONS_00004099targeted PTPRF protein in U87 and U251 cells after lncRNA TCONS_00004099 overexpression and knockdown. ns, not statistically significant; *, $\mathrm{P}<0.05 ;{ }^{* *}, \mathrm{P}<0.01 ;{ }^{* * *}, \mathrm{P}<0.001$. 
viability and proliferation through generating miRNA TCONS_00004099 and targeting PTPRF in glioma cells.

\section{Discussion}

Nervous system neoplasms account for about $3 \%$ of all cancers $(17,18)$, with a mortality rate of 3.4 per 100,000 people (19). Numerous studies have identified non-coding RNAs (ncRNAs) as novel markers in carcinogenesis, among which, lncRNAs appear to be the most promising. Although the most well-known and examined ncRNAs are the miRNAs and it is frequently disrupted in brain tumors (20). However, IncRNA-derived miRNA is much more stable and effective in gliomas, with lncRNAs acting as a repository that is hard to degrade. The function of miRNAs and its interaction with lncRNA in brain tumors have been summarized by different reports (21). miRNAs and lncRNAs were key regulators that mediate tumor growth and oncological behaviors, including tumor proliferation, invasion, apoptosis, and cell metabolism (22). Indeed, lncRNAs have been associated with tumor progression, apoptosis, and cell cycle in gliomas (23). For instance, lncRNA RAMP2-AS1 is remarkably downregulated in GBM and is involved in regulating the cell cycle (24), while lncRNA FOXD2-AS1 promotes glioma progression by regulating cyclin-dependent kinases (25).

Our study shown that the expression of lncRNA TCONS_00004099 was markedly higher in glioma tissues and cell lines compared to normal human brain tissues and normal HEB cell lines. Silencing lncRNA TCONS_00004099 with siRNA limited glioma cell viability, migration, and invasion in U87 and U251 cells, and enhanced apoptosis. Considering the roles of these small non-coding RNAs, novel therapeutic strategies involving miRNA silencing or miRNA mimics may be considered in glioma management.

Our investigation further identified a new miRNA derived from lncRNA TCONS_00004099 and revealed that miRNA TCONS_00004099 increased cell viability and reduced apoptosis in U87 and U251 cells, thereby promoting glioma growth. The miRNA inhibitor significantly suppressed cell migration and invasion, and reversed the effects of lncRNA TCONS_00004099.

Currently, the most common treatment for glioma is maximal surgical resection. Post-surgery radiotherapy and chemotherapy with temozolomide (TMZ) are also first-line treatments for gliomas resulting in higher survival rates in patients (26). However, recurrence and tumor resistance to
TMZ is not uncommon and can lead to treatment failure. In this study, cells were treated with the combination therapy of TMZ $(300 \mu M)$ and downregulation of lncRNA TCONS_00004099. Knockdown of such lncRNA, as well as the miRNA inhibitor both significantly enhanced the TMZ-induced apoptosis compared to the control group. The effects suggested that IncRNA TCONS_00004099 and its associated miRNA may be potential therapeutic targets in combination with TMZ.

Protein Tyrosine Phosphatase Receptor Type F (PTPRF) has a complex role in the regulatory function of cancers. The role of PTPRF in cancer is contradictory, suggesting that PTPRF acts either as an oncogene or as a tumor suppressor in different types of cancer (16,27-32). Recent reports have demonstrated that increased levels of PTPRF are associated with poor prognosis in colorectal cancer patients (14), but the expression and the role of PTPRF and in gliomas still unknown.

Although the relationship between miRNA and PTPRF has been studied (16), but the relationship between PTPRF and lncRNA has so far remained unclear. Thus, in our study, western blot analyses showed that lncRNA TCONS_00004099 was enriched in glioma cells and its overexpression was associated with lower PTPRF expression in both U87 and U251 cells. Interestingly, decreased expression of IncRNA TCONS_00004099 resulted in higher PTPRF expression in U87 and U251 cells, which may represent the complex regulatory role of PTPRF. Nonetheless, xenograft zebrafish models and cell function experiments demonstrated that inhibiting lncRNA TCONS_00004099 blocked tumor proliferation and invasion both in vitro and in vivo. In fact, zebrafish share up to $85 \%$ homology with human genes, and its signal pathway is basically similar to that of human beings. Its biological structure and physiological functions are highly similar to those of mammals and the microenvironment in zebrafish embryo is purer which is suitable for study tumor proliferation and invasion (33). However, the influence of zebrafish gender on the results was not studied in our study, which is also needed to be supplemented in subsequent experiments. Many studies have demonstrated the competitive endogenous RNA (ceRNA) network where lncRNAs act as miRNA sponges to regulate mRNA expression in tumor development. This study, however, described a different type of interaction between lncRNA and miRNA, lncRNA could generate miRNA by RNA splicing and work through miRNA regulation mechanism. miRNA TCONS_00004099 targeted PTPRF mRNA 
and combine with complementary sequences of PTPRF mRNA, inhibit mRNA translation and promote mRNA degradation. The lncRNA TCONS_00004099 promoted cell proliferation and invasion through the derived miRNA (miRNA TCONS_00004099) and its target PTPRF. Significantly, the IncRNA TCONS_00004099/miRNA TCONS_00004099/PTPRF axis may be a critical target for glioma diagnosis and treatment.

\section{Acknowledgments}

Funding: This work was supported by grants from the Guangdong Natural Science Foundation (No. 2020A1515010289); Shenzhen People's Hospital Young and Middle-aged Research Funds Cultivation Fund (No. SYKYPY201923); Shenzhen Key Medical Discipline Construction Fund (Shenzhen Key Medical Discipline 2020-2024); National Natural Science Foundation of China (Award Number: 81602205) and the Young Teacher Training Project of Sun Yat-sen University (Award Number: 20ykpy97).

\section{Footnote}

Reporting Checklist: The authors have completed the ARRIVE reporting checklist. Available at https://dx.doi. org/10.21037/atm-21-2442

Data Sharing Statement: Available at https://dx.doi. org/10.21037/atm-21-2442

Conflicts of Interest: All authors have completed the ICMJE uniform disclosure form (available at https://dx.doi. org/10.21037/atm-21-2442). The authors have no conflicts of interest to declare.

Ethical Statement: The authors are accountable for all aspects of the work in ensuring that questions related to the accuracy or integrity of any part of the work are appropriately investigated and resolved. The study was approved by institutional ethics board of Sun Yat-sen Memorial Hospital, Sun Yat-sen University (No.: 2019060) and conformed to the guidelines of the Declaration of Helsinki (as revised in 2013). Written informed consent was obtained from the patients before the start of the study. Experiments were performed under a project license (No.: LL-KY-2019105) granted by Institute Ethics Committee, Shenzhen People's Hospital, in compliance with China national or institutional guidelines for the care and use of animals.

Open Access Statement: This is an Open Access article distributed in accordance with the Creative Commons Attribution-NonCommercial-NoDerivs 4.0 International License (CC BY-NC-ND 4.0), which permits the noncommercial replication and distribution of the article with the strict proviso that no changes or edits are made and the original work is properly cited (including links to both the formal publication through the relevant DOI and the license). See: https://creativecommons.org/licenses/by-nc-nd/4.0/.

\section{References}

1. Ostrom QT, Bauchet L, Davis FG, et al. The epidemiology of glioma in adults: a "state of the science" review. Neuro Oncol 2014;16:896-913.

2. Krakstad C, Chekenya M. Survival signalling and apoptosis resistance in glioblastomas: opportunities for targeted therapeutics. Mol Cancer 2010;9:135.

3. Jiapaer S, Furuta T, Tanaka S, et al. Potential Strategies Overcoming the Temozolomide Resistance for Glioblastoma. Neurol Med Chir (Tokyo) 2018;58:405-21.

4. Visvader JE, Lindeman GJ. Cancer stem cells: current status and evolving complexities. Cell Stem Cell 2012;10:717-28.

5. Castro-Oropeza R, Melendez-Zajgla J, Maldonado V, et al. The emerging role of lncRNAs in the regulation of cancer stem cells. Cell Oncol (Dordr) 2018;41:585-603.

6. Liao Y, Shen L, Zhao H, et al. LncRNA CASC2 Interacts With miR-181a to Modulate Glioma Growth and Resistance to TMZ Through PTEN Pathway. J Cell Biochem 2017;118:1889-99.

7. Fu PF, Zheng X, Fan X, et al. Role of cytoplasmic lncRNAs in regulating cancer signaling pathways. $\mathrm{J}$ Zhejiang Univ Sci B 2019;20:1-8.

8. Guo JC, Yang YJ, Zheng JF, et al. Silencing of long noncoding RNA HOXA11-AS inhibits the Wnt signaling pathway via the upregulation of HOXA11 and thereby inhibits the proliferation, invasion, and self-renewal of hepatocellular carcinoma stem cells. Exp Mol Med 2019;51:1-20.

9. Bi CL, Liu JF, Zhang MY, et al. LncRNA NEAT1 promotes malignant phenotypes and TMZ resistance in glioblastoma stem cells by regulating let-7g-5p/MAP3K1 axis. Biosci Rep 2020;40:BSR20201111.

10. Tang G, Luo L, Zhang J, et al. IncRNA LINC01057 
promotes mesenchymal differentiation by activating NF-кB signaling in glioblastoma. Cancer Lett 2021;498:152-64.

11. Mu Y, Tang Q, Feng H, et al. lncRNA KTN1-AS1 promotes glioma cell proliferation and invasion by negatively regulating miR-505-3p. Oncol Rep 2020;44:2645-55.

12. Bartel DP. MicroRNAs: genomics, biogenesis, mechanism, and function. Cell 2004;116:281-97.

13. Xue J, Zhou A, Wu Y, et al. miR-182-5p Induced by STAT3 Activation Promotes Glioma Tumorigenesis. Cancer Res 2016;76:4293-304.

14. Gan T, Stevens AT, Xiong X, et al. Inhibition of protein tyrosine phosphatase receptor type F suppresses Wnt signaling in colorectal cancer. Oncogene 2020;39:6789-801.

15. Alonso A, Sasin J, Bottini N, et al. Protein tyrosine phosphatases in the human genome. Cell 2004;117:699-711.

16. Du WW, Fang L, Li M, et al. MicroRNA miR-24 enhances tumor invasion and metastasis by targeting PTPN9 and PTPRF to promote EGF signaling. J Cell Sci 2013;126:1440-53.

17. Mortazavi SMJ, Mortazavi SAR, Paknahad M. Cancers of the Brain and CNS: Global Patterns and Trends in Incidence. J Biomed Phys Eng 2018;8:151-2.

18. Miranda-Filho A, Piñeros M, Soerjomataram I, et al. Cancers of the brain and CNS: global patterns and trends in incidence. Neuro Oncol 2017;19:270-80.

19. GBD 2016 Brain and Other CNS Cancer Collaborators. Global, regional, and national burden of brain and other CNS cancer, 1990-2016: a systematic analysis for the Global Burden of Disease Study 2016. Lancet Neurol 2019;18:376-93.

20. Turner JD, Williamson R, Almefty KK, et al. The many roles of microRNAs in brain tumor biology. Neurosurg Focus 2010;28:E3.

21. Nicoloso MS, Calin GA. MicroRNA involvement in brain tumors: from bench to bedside. Brain Pathol 2008;18:122-9.

22. Gournay M, Paineau M, Archambeau J, et al. Regulat-

Cite this article as: Wang Y, Shan A, Zhou Z, Li W, Xie L, Du B, Lei B. LncRNA TCONS_00004099-derived microRNA regulates oncogenesis through PTPRF in gliomas. Ann Transl Med 2021;9(12):1023. doi: 10.21037/atm-21-2442
INGs in tumors and diseases: Focus on ncRNAs. Cancer Lett 2019;447:66-74.

23. Hanahan D, Weinberg RA. The hallmarks of cancer. Cell 2000;100:57-70.

24. Liu S, Mitra R, Zhao MM, et al. The Potential Roles of Long Noncoding RNAs (lncRNA) in Glioblastoma Development. Mol Cancer Ther 2016;15:2977-86.

25. Wang J, Li B, Wang C, et al. Long noncoding RNA FOXD2-AS1 promotes glioma cell cycle progression and proliferation through the FOXD2-AS1/miR-31/CDK1 pathway. J Cell Biochem 2019;120:19784-95.

26. Weller M, van den Bent M, Tonn JC, et al. European Association for Neuro-Oncology (EANO) guideline on the diagnosis and treatment of adult astrocytic and oligodendroglial gliomas. Lancet Oncol 2017;18:e315-29.

27. Wang X, Liu T, Bai Y, et al. Polymerase I and transcript release factor acts as an essential modulator of glioblastoma chemoresistance. PLoS One 2014;9:e93439.

28. Nunes-Xavier CE, Martín-Pérez J, Elson A, et al. Protein tyrosine phosphatases as novel targets in breast cancer therapy. Biochim Biophys Acta 2013;1836:211-26.

29. Levea CM, Mcgary CT, Symons JR, et al. PTP LAR expression compared to prognostic indices in metastatic and non-metastatic breast cancer. Breast Cancer Res Treat 2000;64:221-8.

30. Wang Z, Shen D, Parsons DW, et al. Mutational analysis of the tyrosine phosphatome in colorectal cancers. Science 2004;304:1164-6.

31. Bera R, Chiou CY, Yu MC, et al. Functional genomics identified a novel protein tyrosine phosphatase receptor type F-mediated growth inhibition in hepatocarcinogenesis. Hepatology 2014;59:2238-50.

32. DaSilva JO, Amorino GP, Casarez EV, et al. Neuroendocrine-derived peptides promote prostate cancer cell survival through activation of IGF-1R signaling. Prostate 2013;73:801-12.

33. Osmani N, Goetz JG. Multiscale Imaging of Metastasis in Zebrafish. Trends Cancer 2019;5:766-78. 
The sequence of LncRNA TCONS_00004099 and the derived microRNA

>TCONS_00004099 gene=RP11-418J17.1

TTCGAAGGTCGCAATGATTTGTATGACAGCTGCTAGCCCAAGAGAGAGTTCCAGAAGAGGGCTACCTAACA GGTGCTGTGGCATTTAGAAGAGGAATGGAGCCATTGTCAACGCACAGCTTGCAGGGGAGCAGGAGAGTC AGTCTAAGAAGGAAATGTGATGATGGAAACAGAGGTTGGAAGATGGAAGAGGACCCAGAGTCAAGGAATGC AGGTAGAAAAGAAAAGCCTGGATTCTCCCCTAAAGCCTCCAGATGGAACACAGCCCTGCCTCCACCTTGAT TTCAGGACTTCTTACCCCAAGAATCGTGTCTGTCTTCATCCTAAGGAGTCAACTGCTATACCTTAGTGATT TCAATGTAATTGTTTGGTCTGTAAGTCATTCTTGCCACTGTGGTTCTGGGGCCATTGTTCTGCAACGAT GAATAAACAATGTTTTAGAAATCACTCATCCAAGAAAGGTGACAAGATACTGGAATAGAGGTATTTGAAAAAT ATTCATGGGAATTTTAGAGGATTGTAGATGGTCTGAATACCTGTGAAGACATCATCTAATGTATCTAAAAAC CACATAATGCTTCATCTCAATGTATTTTAAGAATTAATTTTGTTATAAGCTAGAATTTTTCATTTTGGTTTTA ATAATAACATCAAAAGGATTTTTTAACATAAATTAGAAAGGGCATACATGGAGAAGTAAAGAAGAATTCTTCTT GGGATTAGCCAAAAATTAGTGAGAGATTACATATTTCCTTAGATGAAAAATCCTACAGGCAAACTTTTAATGT CAGGGAGAGGAATACTTTATTACCTAATAATGTAGATGAAAAGAATAGCTAAGCATTCTTTTTTAAAGCAGTA TACAGAAATGAGGGAAAGGCTAAGAGGTTAACTCATTTCTATTTTGAACTCTATTTTTTTTAAAACCCTTGC TCCAAATTTTACTTACTATCTTTTAAATGTCATCCTGCTAGAGTGTAAGTCTTTAGTTTTAGTTGCTTAATA AAAATTAATTTTTTGTGACAAAAGGCA

>pre-miRNA

uucuugccacUGUGGUUCUGGGGCCAUUGUUcugcaacgaugaauaaacaauguuuuagaaaucacucauccaagaaag

$>$ miRNA

UGUGGUUCUGGGGCCAUUGUU 